* Fokke Tuinstra ${ }^{1}$ and Bouke F. Tuinstra ${ }^{2}$

* ' Delft University of Technology, Delft, The Netherlands

-E-mail: f.tuinstra@tudelft.nl

${ }^{2}$ Stork FDO Inoteq b.v., Amsterdam, The Netherlands

•E-mail: bouke.tuinstra@stork.com

DOI: $10.1051 /$ epn/2010302

\title{
THE WEIGHT OF
}

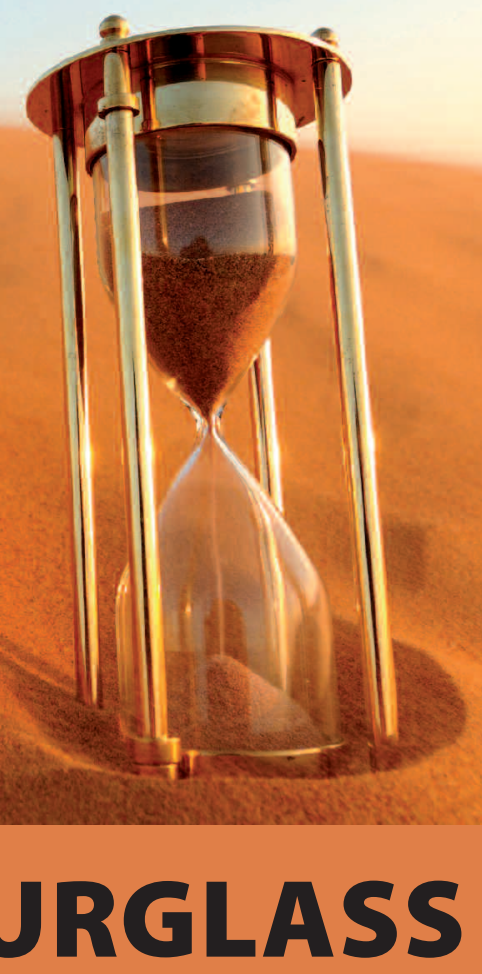

\section{It is a favourite question in science quizzes: Does an hourglass in operation weigh less or more than in the static state? The 'airborne' part of the sand makes it lighter, and the impact of the sand hitting the bottom makes it heavier. The classical question is: "Do they compensate?" But it is more interesting than that, as a thorough scientific approach will show.}

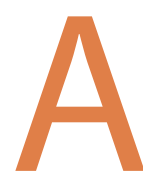

special feature of an hourglass - or egg timer - is that the mass flow $\dot{\mathrm{m}}[\mathrm{kg} / \mathrm{s}]$ of sand through the narrow orifice is fairly independent of the sand level. This is caused by the granular nature of the sand. The flow $\mathrm{m}$ is present in the free-falling stream of sand but also in the slowly moving dense mass in the upper compartment. If the free-fall time of the sand is $\tau$, the weight of the free-falling mass is g. $(\dot{m} \tau)$. When hitting the surface in the lower compartment, it exerts a momentum per second, i.e., a downward force $(\mathrm{g \tau})$ on the surface. This force exactly compensates the missing weight of the sand that is in free fall. This was the beautiful argument to conclude that the weight should stay the same. However, in 1985 Shen and Scott [1] showed that the centre of mass (c.o.m.) of the sand is not moving down at constant speed, but is decelerating during the steady operation of an hourglass. That causes a subtle gain of its weight on a balance. With an ingeniously constructed hourglass they even managed to confirm the presence of excess weight. However, with their set-up it was not possible to measure the effect in a quantitative way.

We have designed a multiple-orifice set-up that can be used for quantitative measurements using state-of-the art laboratory weighing equipment. We will show that the theoretical result can be confirmed by measurements. Furthermore, we will present a more thorough analysis of the motion of sand in an hourglass that allows us to understand the mechanism behind the increase in weight.

\section{Hundreds of holes}

The set-up we used is shown in figure 1. It allows us to accurately measure the weight increase, even with a standard balance. The hourglass has two special features. a. The upper and lower compartments have an identical cylindrical shape. This has the advantage that the weight effect to be measured is constant during the discharge of the hourglass.

b. The single orifice in a standard hourglass has been replaced by a plate containing a few hundred identical holes, which we will call the sieve. This suppresses the noise and increases the mass flow which amplifies the effect we are looking for.

In the approach of Shen and Scott [1] the deceleration of the centre of mass of the sand is presented in a general, though complex, formula. For the cylindrical shape the calculation is reduced to an elementary exercise in 
III statics. The z-coordinate of the c.o.m. of all of the sand in the hourglass is found to be:

$$
\mathrm{z}_{\mathrm{com}}=\frac{1}{\mathrm{M}}\left[\mathrm{z}_{u} \cdot \mathrm{m}_{u}+\mathrm{z}_{l} \cdot \mathrm{m}_{l}\right]
$$

where $\mathrm{M}$ is the total mass and the indices $u$ and $l$ stand for upper and lower, respectively. The mass that is in free-fall does not show up here. The argument from the introduction tells us that its contribution can be considered to be accounted for in $\mathrm{m}_{l}\left(=\mathrm{M}-\mathrm{m}_{u}\right)$.

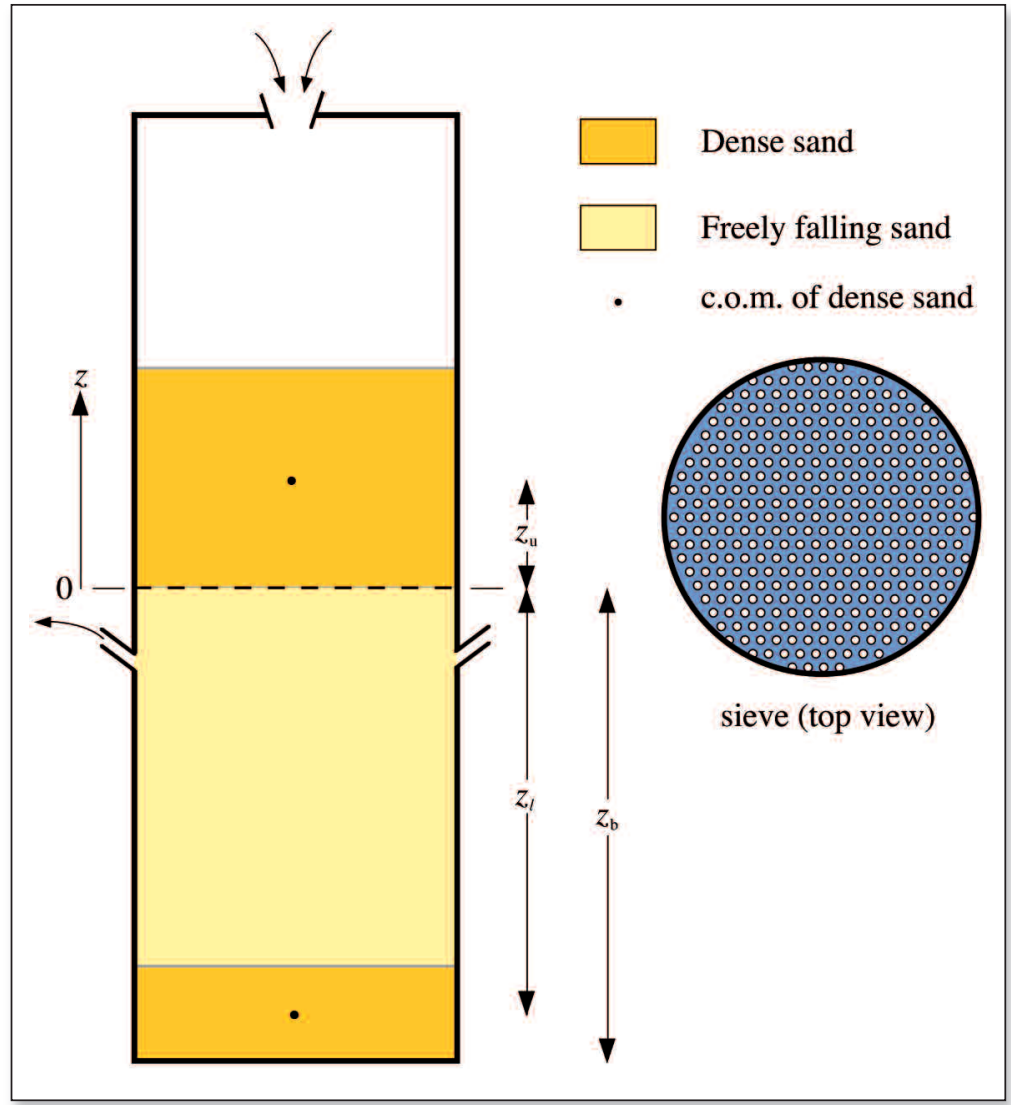

$\Delta$ FIG. 1: Design of the cylindrical multiple hourglass. The initial height of the sand column in the upper compartment is $\mathrm{L}$ (i.e., $\mathrm{z}=\mathrm{L}$ ). The top level is descending at a constant speed of $L / T$. $T$ is the time at which $z=0$. The sand level in the lower compartment is rising at the same speed. The total sand mass is $\mathrm{M}$. The sand levels in both compartments stay horizontal and flat. $z_{b}$ is the height of the lower compartment. The positions of the c.o.m. of all of the sand in the upper and lower compartment are given by $z_{u}$ and $z$ respectively. The operation is started by removing an aluminium sheet which initially is located directly underneath the sieve. Both compartments are vented.

Since $\mathrm{z}_{u}, \mathrm{z}_{l}, \mathrm{~m}_{u}$ and $\mathrm{m}_{l}$ all change linearly with time, their products appearing in $\mathrm{z}_{\mathrm{com}}$ change quadratically. Therefore, the second time derivative of $\mathrm{z}_{\mathrm{com}}$ is not zero but independent of the time $t$ and one finds a constant downward force:

$$
\mathrm{F}=\frac{2 \mathrm{M} \cdot \mathrm{L}}{\mathrm{T}^{2}}
$$

The cylindrical form is not the crucial step that makes a quantitative experiment possible, but it is, first of all, the large number of holes. When a standard hourglass is placed on a balance, it will be quickly observed that during operation its weight is far from steady. There are several sources for excessive noise. The most conspicuous source is formed by the uncontrolled avalanches occurring on the conical slopes in both the upper and the lower compartment. A second source of instabilities is the fluctuating gas pressure difference across the orifice. This temporarily blocks the orifice by stabilizing arches of sand. When enough gas has seeped upward to equalise the pressure, the formed domes will collapse. $[2,3,4]$. These domes of sand continuously form and break down. This can even cause periodic oscillations in the flow [4]. Both effects are avoided in the design used here.

\section{The experiment}

The hourglass consisted of two polycarbonate cylinders, each having a diameter of $8 \mathrm{~cm}$ and a length of $20 \mathrm{~cm}$. They were cut out of soda bottles. Mounted on top of each other they were separated by a flat plate as shown in figure 1. In this plate, 230 holes with a diameter of $2.0 \mathrm{~mm}$ were drilled (the 'sieve'). Initially, the holes are blocked by an aluminium plate. Ordinary silver sand from a garden centre was used to fill the hourglass. Large grains were removed by sieving them out. The maximum diameter of the grains was $0.2 \mathrm{~mm}$, so the expected flow is well within the granular flow regime [3]. After filling the upper compartment with one litre of sand $(\approx 1.6 \mathrm{~kg})$ the setup was mounted on a balance which could weigh up to $2 \mathrm{~kg}$ with an accuracy of 0.01 gram $\left(10^{-4} \mathrm{~N}\right)$. To start the measurement, the aluminium plate is removed manually.

We first determined the sand flux through the sieve, collecting the sand during a discharge of the upper compartment. The sand flow was $\dot{\mathrm{m}}=(\mathrm{M} / \mathrm{T})=67.8 \pm$ $0.8 \mathrm{~g} / \mathrm{s}$ and constant as expected (see figure 2).

We also determined the constant speed $\mathrm{v}$ of the descending top level of the sand: $\mathrm{v}=(\mathrm{L} / \mathrm{T})=0.84 \pm 0.06 \mathrm{~cm} / \mathrm{s}$. According to formula (1) the extra force should then be $2 \mathrm{~m} . \mathrm{v}=1.14 \pm 0.10 \times 10^{-3} \mathrm{~N}$, (equivalent to $0.116 \pm 0.010$ gram). During the discharge of the hourglass the balance showed an excess reading of $0.121 \pm 0.006$ gram, which is in excellent agreement with what we would expect. (see figure 3).

At the end of the discharge a hump is observed. It is due to the last bit of sand in free fall. Its presence and actual size will be discussed in the next section. After the discharge we observed that some very fine sand dust had settled around the setup, apparently escaped through the ventilation openings. This may be the reason of the slightly negative slope during the discharge.

The resulting additional weight is a rather small fraction of the weight of the total sand mass $M$.

For a classroom demonstration a more substantial effect would be more convincing. This may be achieved by broaching the holes. An extension of the diameter of the orifices from $2 \mathrm{~mm}$ to $3 \mathrm{~mm}$ would, according to the theory and experiment $[2,5]$, shorten $\mathrm{T}$ by a factor $(1.5)^{-2.5}=0.36$, thus increasing the excess weight to about $10^{-2} \mathrm{~N}$ (or 1 gram of mass). So a much less delicate 
balance could be used. The reduction of time $\mathrm{T}$ to $7 \mathrm{~s}$ would still allow an accurate weight measurement.

\section{Taming the free fall}

The calculation of the motion of the c.o.m to arrive at equation (1) leads us to the correct answer but fails to give us any insight in the underlying physics. We therefore prefer to follow the changing momentum of the sand and look at the corresponding force diagram.

Imagine in our experiment we forgot to insert the sieve. On removing the aluminium sheet, the whole sand mass $M$ would drop down at once. The balance would have a rough ride, its static reading would be disturbed by a sudden oscillation, as sketched in figure 4. The momentum starting at zero will return to zero at the end of the process. For the force diagram, being the time derivative of the momentum, this implies that the surface area of the negative part and the positive part of the oscillation should be the same.

In the actual hourglass, the sieve is an obstruction which impedes the free fall into a slow controlled motion.

Above the sieve a dense sand mass is slowly moving downward at constant speed, below it a small fraction of the mass is speeding up in free fall. The orifice is, metaphorically speaking, causing a traffic jam. As on a motorway, after slowly having passed the site of the accident, the traffic density decreases dramatically as it is speeding up. The cause is simple: in a stationary situation, the number of cars per second passing any site along the road will be the same. The higher the speed the lower the density. The same applies to any stationary single flow of matter, like the sand in the hourglass. Along the stream the mass per length unit $\rho^{\prime}[\mathrm{kg} / \mathrm{m}]$ is inversely proportional to the speed $\mathrm{v}$. The momentum per unit length $\rho^{\prime} \mathrm{v}$ is constant. It is equal to the fixed flux $\dot{\mathrm{m}}$, which is limited by the obstruction. The total momentum of such a stream thus is m times its length, whatever changes in the cross section or speed may occur. If the top levels in both compartments in the hourglass are flat, the momentum of the moving sand is $\dot{\mathrm{m}}$ times the distance between these levels. And the force is the rate of change of that distance. The rough freefall has been tamed by the obstruction and controlled by the constant flux $\dot{m}$ it allows to pass.

If we take the orifice - or in our case the sieve - as the reference height, and call the distance from the orifice the upper and lower levels $\mathrm{L}_{\mathrm{u}}$ and $\mathrm{L}_{l}$ respectively, the total momentum is $\mathrm{p}=\dot{\mathrm{m}}\left(\mathrm{L}_{\mathrm{u}}+\mathrm{L}_{l}\right)$, rendering a force

$$
\mathrm{F}=-\dot{\mathrm{m}}\left(\frac{\mathrm{dL}_{\mathrm{u}}}{\mathrm{dt}}+\frac{\mathrm{dL}_{\mathrm{l}}}{\mathrm{dt}}\right) \text {. }
$$

Apparently the only terms that matter are the speeds of the moving lower and upper sand levels. Watching a discharging hourglass we note that absolute rest exists below the lower level and above the upper one and in addition in between both levels an absolute stationary

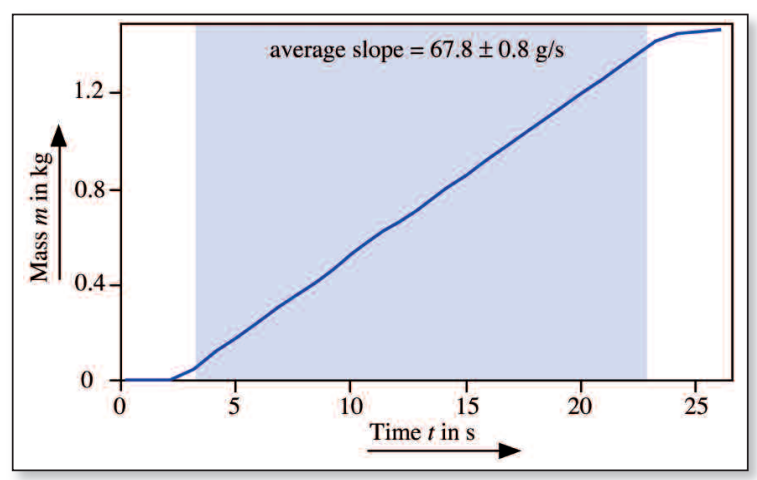

state is observed. So indeed the only physical changes in the overall state are the positions of the sand levels $\mathrm{L}_{\mathrm{u}}$ and $\mathrm{L}_{l}$.

It is the change of these levels themselves giving rise to a loss of momentum per second. In our cylindrical setup both rates are equal to $L / T$ and since $\dot{m}=M / T$, we obtain formula (1).

Figure 5 shows the momenta and the corresponding forces as a function of time. The momenta of both terms are tapering off linearly from $\mathrm{ML} / \mathrm{T}$ to zero at time $\mathrm{T}$. The resulting force is constant.

Compared with figure 4 , the narrow positive crash peak is now smeared out over time T, which is orders of magnitude larger than the duration of the crash.

\section{What happened to the initial dip?}

Before and after the discharge, all of the sand is at rest. The blue momentum curve should drop from zero at $\mathrm{t}$ $=0$ to the value $-2 \mathrm{~L} \times \dot{\mathrm{m}}$. The drop cannot be instantaneous. The length of the sand stream that acquires momentum is increasing during a short period $\tau$, ending when the cascade hits the floor of the lower compartment. It causes a very short but strong dip in the force diagram. As in the force diagram of figure 4 , the surface area of this dip must be equal to the positive part of the diagram, though it is compressed to a pulse. In our experiment this pulse is completely swamped by the forces needed to remove the aluminium sheet. For this reason we had to discard the first few seconds in figure 3. As a result, the throughput time $\mathrm{T}$ can not be determined with acceptable accuracy. Figure 5 does not predict the hump at the end observed in the experiment (fig.3). In the ideal setup, the lower compartment should be filled precisely up to the sieve

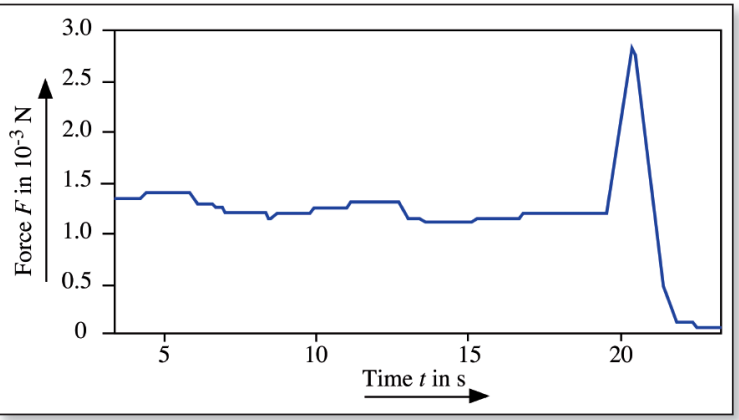

4 FIG. 2:

Mass of sand collected during discharge of the top compartment through the sieve into a freestanding bowl.
4FIG. 3: Results of an experiment with the multiple hourglass: the additional force exerted by the running hourglass on the balance. 
III when the upper compartment gets empty at $t=\mathrm{T}$. In practice this is hard to achieve. A small gap $\Delta \mathrm{L}$ between the final sand level and the sieve is unavoidable. In our experiments the gap was smaller than $1 \mathrm{~cm}$. The last bit of sand falling as the upper compartment is empty reduces the length of the sand stream suddenly with the speed of fall. For $\Delta \mathrm{L}<1 \mathrm{~cm}$, this would result in a surface area of a hump to be less than $\Delta \mathrm{Lm} \approx 0.007 \mathrm{Ns}$. The area observed in fig 3 is of the order of $0.004 \mathrm{Ns}$, suggesting that the gap effectively is about half a centimeter wide.

Formula (1) is not exact because of the starting pulse and the hump at the end. Both these effects, however, take times in the order of only a few tenths of a second; negligible compared with $\mathrm{T}$, which is more than 10 seconds.

\section{Physics of the flowing sand}

The only active region in an hourglass lies between the sand levels in the two compartments. The rest of the sand is dead weight. The amount of sand in motion is constantly decreasing as follows from the continuously decreasing distance between the two sand levels. At the lower level a mass m per second is transferred from the moving mass to the dead weight. So that is the place where the momentum is annihilated and consequently that is the place where the extra force is exerted.

The eye-catching tiny cascade in an hourglass seems not to contribute to the extra weight. Why don't we see

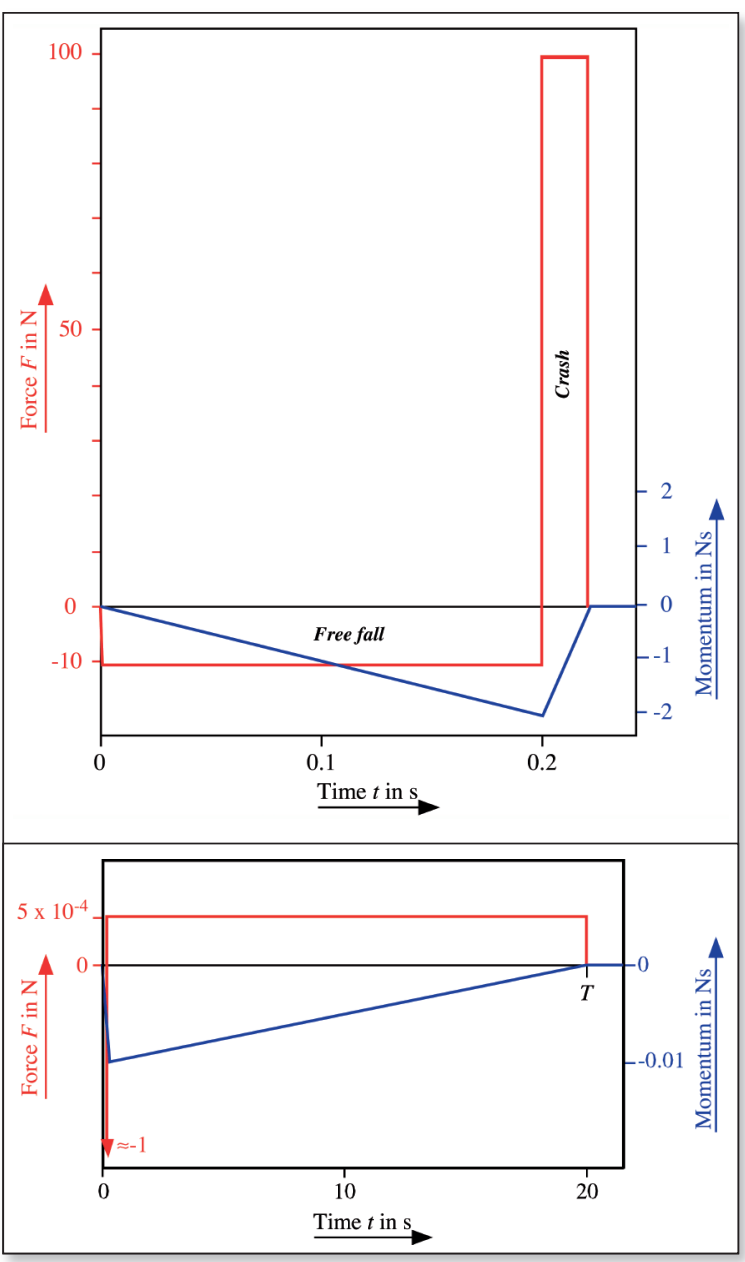

any effect of it in the theoretical results, where only the speed of the two sand levels seems to matter? Doesn't the speed of the falling sand in the cascade contribute to the momentum? It sure does, but in exactly the same way as the slow moving sand in the upper compartment. At any place along the flowing sand, the contribution to the momentum is exactly m per meter. The role of the cascade is not different from the slow moving compact sand above the sieve. This corresponds to the idea put forward in the introduction that the impact of freely falling sand compensates for its weightlessness. We now see that this cannot be exactly true. The reason is that the free falling sand enters the cascade at a finite speed while at the same time the surface of the lower compartment rises with a finite speed to meet it at the lower end.

In the old days it was a miraculous practice of some milkmen to pour milk from a jug at an appreciable height into a pot on a balance. If they stopped pouring exactly at the moment that the pointer on the scale reached the one kilogram mark, the final content collected in the pot was exactly one kg (that is, one liter), regardless of the height they were pouring from. We now understand that this is only approximately true, but it certainly was good practice in the dairy business.

\section{About the authors}

Fokke and Bouke Tuinstra (father and son) both received their PhD at Delft University of Technology. Fokke is professor emeritus of the TU-Delft where he taught solid-state and general physics. His research comprises the molecular structure of sulphur, Raman spectroscopy of graphite, incommensurate crystals and surface phenomena.

Bouke is a chemical engineer working in product and technology development in the field of energy conversion. His primary scientific interests are in modeling of materials and processes involving the transport of heat and matter.

\section{Acknowledgement}

We would like to thank J.M.H.M. van Veen for his assistance with the measurements.

\section{References}

[1] K.Y. Shen and B.L. Scott (1985), Am. J. Phys. 53, 787.

[2] R.M. Nedderman, Statics and Kinematics of Granular Materials, Cambridge University Press, Cambridge (1992).

[3] X. Wu, K.J. Måløy, A. Hansen, M Ammi and D. Bideau (1993), Phys. Rev. Lett. 71, 1363.

[4] B.K. Muite, M.L. Hunt and G.G. Joseph (2004), Phys. of Fluids 9, 3415.

[5] J. Flores, G. Solovey \& S. Gil (2003), Am. J. Phys 71, 715.

[6] V. Becker and T. Pörschel (2008), Granular Matter 10, 231. 